\title{
Editorial
}

\section{Control Problem of Nonlinear Systems with Applications}

\author{
Rongwei Guo, ${ }^{1}$ H. G. Enjieu Kadji, ${ }^{2}$ Xinguang Zhang, ${ }^{3}$ \\ Uchechukwu E. Vincent, ${ }^{4}$ and Wenguang $\mathbf{Y u}^{5}$ \\ ${ }^{1}$ School of Science, Qilu University of Technology, Jinan 250353, China \\ ${ }^{2}$ Center for Neural Engineering, The Pennsylvania State University, University Park, PA 16802, USA \\ ${ }^{3}$ Department of Mathematics and Statistics, Curtin University of Technology, Perth, WA 6845, Australia \\ ${ }^{4}$ Department of Physical Sciences, Redeemer's University, PMB 230, Ede, Nigeria \\ ${ }^{5}$ School of Insurance, Shandong University of Finance and Economics, Jinan 250014, China
}

Correspondence should be addressed to Rongwei Guo; rongwei_guo@163.com

Received 29 June 2016; Accepted 17 July 2016

Copyright (C) 2016 Rongwei Guo et al. This is an open access article distributed under the Creative Commons Attribution License, which permits unrestricted use, distribution, and reproduction in any medium, provided the original work is properly cited.

The control problem of nonlinear systems with applications is general in the actual process and has attracted many scholars' attention owing to the wide applications in various fields such as physics, mathematics, finance, and engineering. Therefore, the analysis and synthesis of control problems play important roles in many practical systems. The aim of this special issue is to bring together the latest/innovative knowledge, analysis, and synthesis of control problems of nonlinear systems. We publish in this issue a number of state-of-the-art studies on the topic that span the control problems in economics, nonlinear control problems, nonlinear stability problems, and control problems in chaotic systems.

For control problems in economics, W. Yu and Y. Huang investigate a dependent insurance risk model with surrender and investment under the thinning process. By the martingale theory, the properties of the surplus process, adjustment coefficient equation, the upper bound of ruin probability, and explicit expression of ruin probability are obtained. X. Zhou et al. provide a practical optimal reinsurance scheme under particular conditions, with the goal of minimizing total insurer risk, and explore the optimization of limited stop-loss reinsurance under three risk measures: value at risk (VaR), tail value at risk (TVaR), and conditional tail expectation (CTE). Q. Liu et al. consider the decomposition and decoupling analysis of energy-related carbon emissions from China manufacturing. L. Xu et al. study the optimal investment and consumption for an insurer with highwatermark performance fee and the object of insurance company is to maximize the expected cumulated discount utility up to ruin time. C. Chen et al. establish a posteriori error estimate for finite volume element method of a second-order hyperbolic equation. Residual-type a posteriori error estimator is derived. The computable upper and lower bounds on the error in the $H_{1}$-norm are established. Numerical experiments are provided to illustrate the performance of the proposed estimator. H.-L. To et al. present a cascade probability control scheme using margin optimal method to address such challenges under different kinds of real-world TCP stacks. Simulation results guarantee the measured round trip time tracking to a low value of delay.

On nonlinear control problems, M.-D. Tran and H.J. Kang present a high-performance nonsingular terminal sliding mode control method for uncertain second-order nonlinear systems. Y. Abe et al. propose a systematic numerical method for designing robust nonlinear $H_{\infty}$ controllers without a priori low-dimensional approximation with respect to solutions of Hamilton-Jacobi equations. J. Yu et al. study the global asymptotic stabilization control problem for a class of nonlinear systems with input-to-state stable (ISS) dynamic uncertainties and uncertain time-varying control coefficients. Z. Wang and Z. Yan consider a three-stage tracking control for the LED wafer transporting robot and obtain a high order polynomial interpolation method to plan the motion process of the LED wafer transporting robot. D. I. R. Almeida et al. designed a strategy and implemented a robust controller for a class of underactuated mechanical systems, with two degrees of freedom, which solves the problems of regulation and trajectory tracking. 
L. Li et al. apply an unsupervised learning algorithm to learn language-independent stroke feature and combine unsupervised stroke feature learning together with automatically multilayer feature extraction to improve the representational power of text feature and develop a novel nonlinear network based on traditional Convolutional Neural Network that enables detecting multilingual text regions in the images. Y.-C. Wang et al. present a unified approach to nonlinear dynamic inversion control algorithm with the parameters for desired dynamics determined by using an eigenvalue assignment method. F. A. G. Becerra et al. investigate the optimal controller and controller based on differential flatness in a linear guide system: a performance comparison of indexes. $Z$. Yao et al. studied the coordinated stability control of windthermal hybrid AC/DC power system. A coordinated control strategy for the wind-thermal hybrid AC/DC power system is proposed and an experimental prototype is made. Z.-R. Tsai and Y.-Z. Chang propose an adaptive control scheme for nonlinear systems with significant nonminimum phase dynamics. The scheme is composed of an inner-level adaptive fuzzy PD control law and an outer-level supervisory control law. C. He et al. address the development and application of novel Prognostics and Health Management (PHM) technologies to a prototype machining process (a screw tightening machine). The enabling technologies are built upon a series of tasks starting with failure analysis, testing, and data processing aimed at extracting useful features or condition indicators from raw data, a symbolic regression modeling framework, and a Bayesian estimation method called particle filtering to predict the feature state estimate accurately. $\mathrm{H}$. Lou et al. propose a novel visual tracking algorithm based on multifeature selection and sparse representation in order to enhance the robustness of visual tracking algorithm in complex environment. E. Kolsi-Gdoura et al. consider the surface design as a case of virtual controller design using the back-stepping method. P. Jiang et al. study the multivariable fuzzy control based mobile robot odor source localization via semitensor product. $\mathrm{Z}$. Wu et al. present an adaptive neural control for the longitudinal dynamics of a morphing aircraft. W. Sun et al. present $H_{\infty}$ excitation control design problem for power systems with input time delay and disturbances by using nonlinear Hamiltonian system theory. S. ud Din et al. present a robust control design for the class of underactuated uncertain nonlinear systems. C. Gong et al. researched on submarine straight-line track control underwater base on nonlinear proportion differential.. M. C. Razali et al. studied the singularly perturbation method which is applied to multivariable PID controller design, found that the singularly perturbed system obtained by Naidu method can maintain the originality of the system characteristics, and then designed MPID controllers. It should be pointed out that the closed loop performance and process interactions were analyzed and compared to see the effectiveness of the singularly perturbed MPID control design.

On nonlinear stability problems, J. Chen investigates the generalized Degasperis-Procesi equation with variable coefficients and establishes the $L_{1}(\mathrm{R})$ stability of the strong solution for the equation under certain assumptions. J. A. Taborda and F. Angulo describe and prove a new method to compute and control the basins of attraction in multistability scenarios and guarantee monostability condition. In particular, the basins of attraction are computed only using a submap, and the coexistence of periodic solutions is controlled through fixed-point inducting control technique, which has been successfully used until now to stabilize unstable periodic orbits. D. Xu et al. discuss the global dynamics of a model involving an endemic equilibrium and a disease-free equilibrium, respectively. V. Nosov et al. analyze the stability of autonomous dynamical switched systems by means of multiple Lyapunov functions and give the stability theorems which have finite number of conditions to check. Y. Tian et al. address the problem of exponential stabilization of a class of time-varying delay systems with nonlinear perturbations. J. Liu and Y. Yang investigated the limiting distribution of the size of binary interval tree. M. De la Sen and A. Ibeas investigated some boundedness and convergence properties of sequences which are generated iteratively through switched mappings defined on probabilistic metric spaces as well as conditions of existence and uniqueness of fixed points. Such switching mappings are built from a set of primary self-mappings selected through switching laws. $\mathrm{H}$. $\mathrm{Xu}$ et al. studied the $L_{2}$-gain analysis problem for a class of discrete-time switched systems with time-varying delays. A mode-dependent average dwell time (MDADT) approach is applied to analyze the $L_{2}$-gain performance for these discretetime switched delay systems. H. Zhu et al. study the flow past a pair of cylinders in tandem at Reynolds numbers of 1000 by Domain Decomposition Method by applying a parallel computation.

Four papers on control problems in chaotic systems have been published. T. H. Cortés et al. studied the complete synchronization and the generalized synchronization problem of the discrete-time chaotic fuzzy systems by means of fuzzy output regulation using genetic algorithm. L. Ren and R. Guo investigated the synchronization and antisynchronization for a class of chaotic systems, and not only proposed a necessary and sufficient condition to synchronize and antisynchronize simultaneously the chaotic systems but also obtained two methods to realize coexistence of synchronization and antisynchronization in the chaotic systems, and give the corresponding adaptive controllers. Z. Li et al. studied chaotification problem for a class of delay difference equations by using the snap-back repeller theory and the feedback control approach. L. Huang et al. investigate the design and application in secure communication and image encryption of a new Lorenz-like system with varying parameter. L. Huang et al. proposed a new Lorenz-like chaotic system with varying parameter by adding a state feedback function.

We hope these papers will be of help to readers in furthering their exploratory research on control problem of nonlinear systems with applications and related topics.

Rongwei Guo

H. G. Enjieu Kadji Xinguang Zhang

Uchechukwu E. Vincent Wenguang $Y u$ 


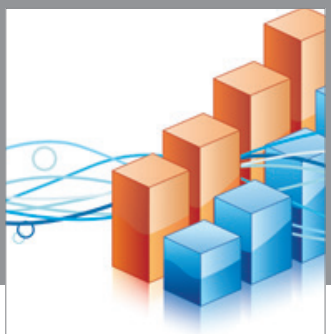

Advances in

Operations Research

vatem alat4

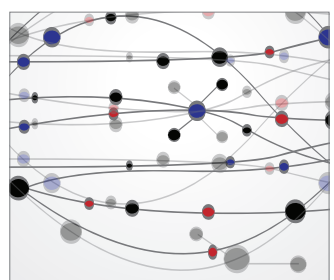

\section{The Scientific} World Journal
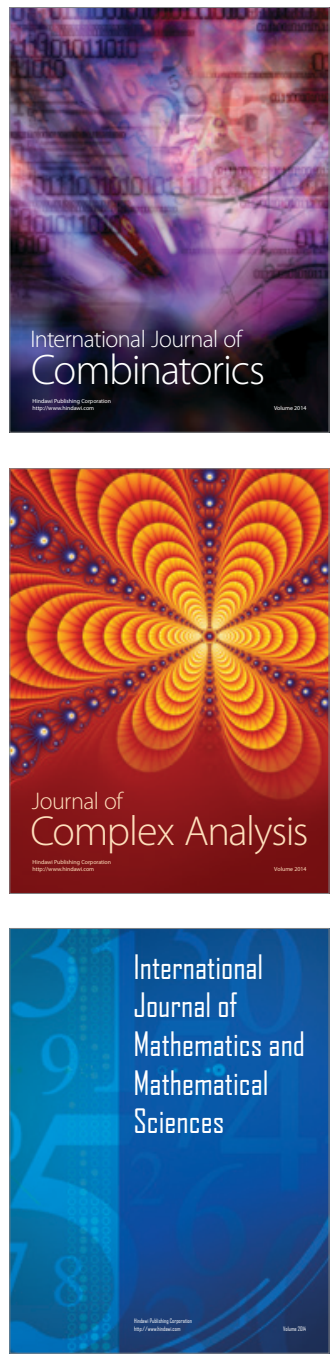
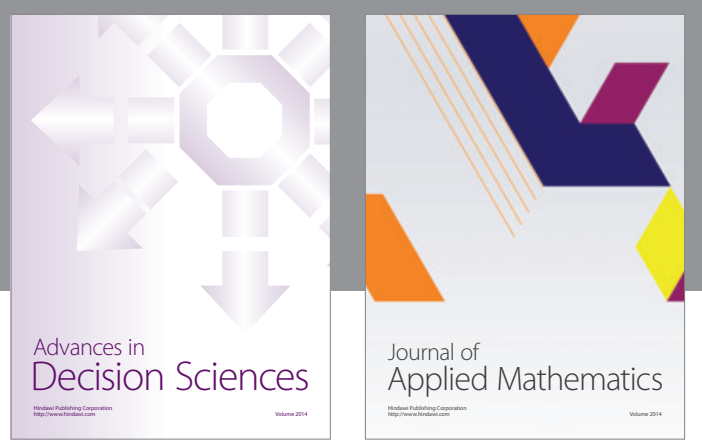

Algebra

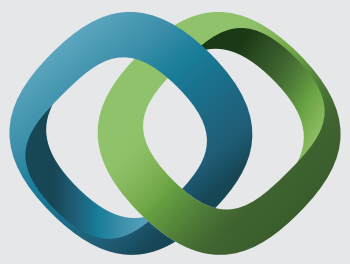

\section{Hindawi}

Submit your manuscripts at

http://www.hindawi.com
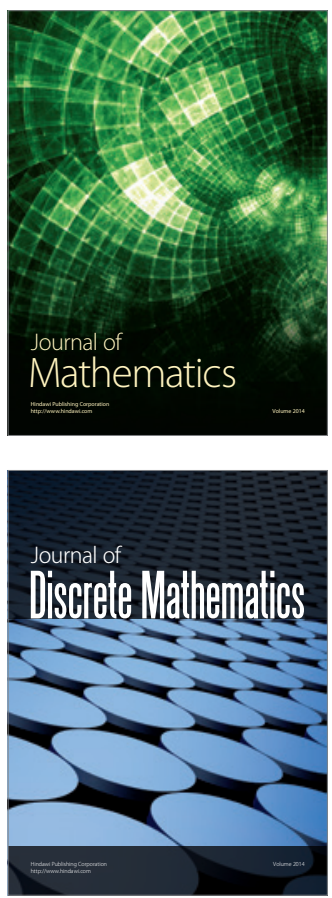

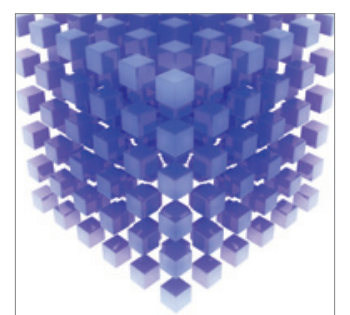

Mathematical Problems in Engineering
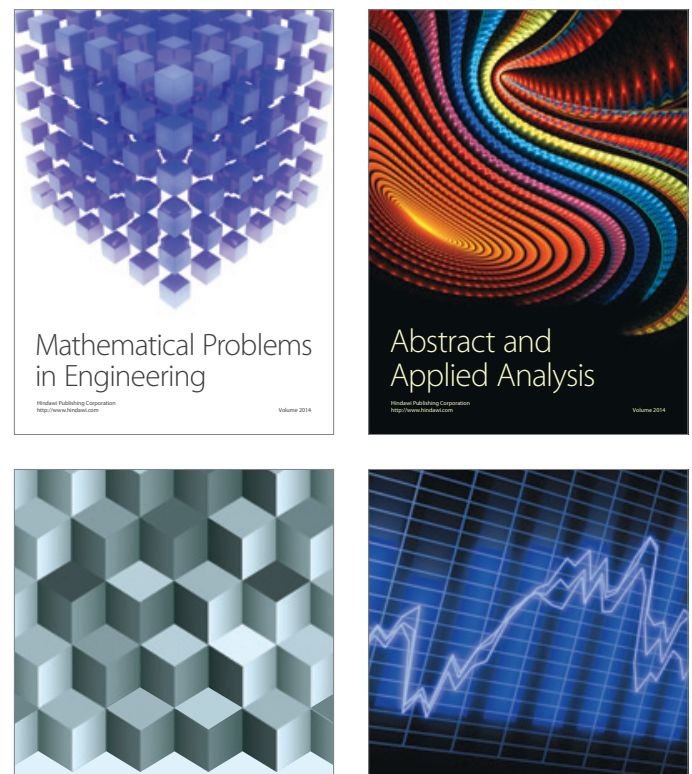

Journal of

Function Spaces

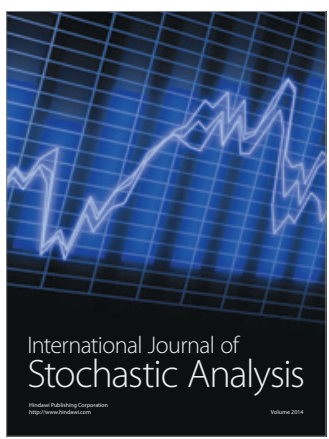

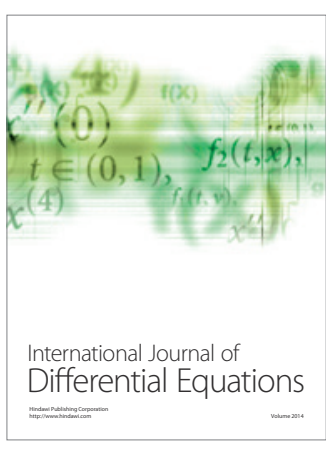
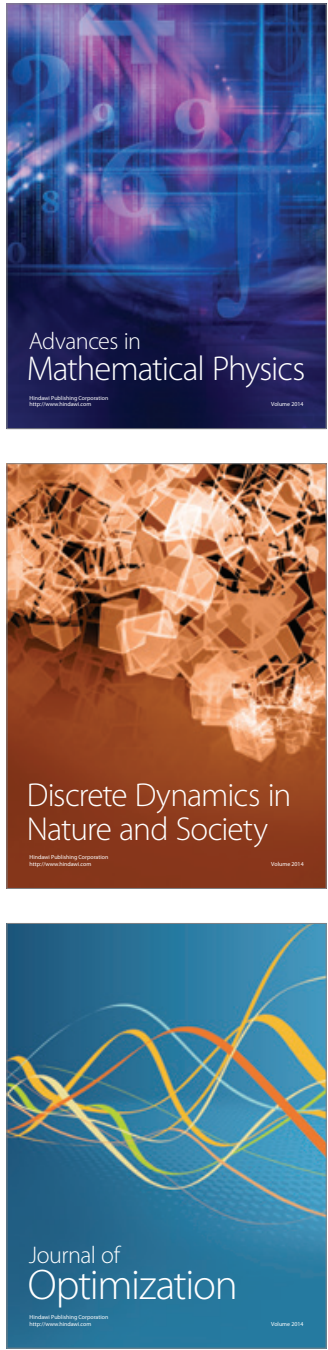